\title{
Isolation and characterization of lipid-degrading bacteria in wastewater of food processing plants and restaurants in Can Tho city, Vietnam
}

\author{
Ngo Thanh Phong ${ }^{1, *}$, Nguyen Thanh Duyen ${ }^{2}$, Cao Ngoc Diep ${ }^{3}$ \\ ${ }^{1}$ Dept. of Biology, College of Natural Sciences, Can Tho University, Can Tho City, Vietnam \\ ${ }^{2}$ Center for Continuing Education of Cairang district, Can Tho city, Vietnam \\ ${ }^{3}$ Dept. Microbiology Biotechnology, Biotechnology R\&D Institute, Can Tho University, Can Tho City, Vietnam
}

Email address:

ngophong@ctu.edu.vn (N. T. Phong), nguyenthanhduyen1983@yahoo.com.vn (N. T. Duyen),cndiep@ctu.edu.vn (C. N. Diep)

\section{To cite this article:}

Ngo Thanh Phong, Nguyen Thanh Duyen, Cao Ngoc Diep. Isolation and Characterization of Lipid-Degrading Bacteria in Wastewater of Food Processing Plants and Restaurants in Can Tho City, Vietnam. American Journal of Life Sciences. Vol. 2, No. 6, 2014, pp. $382-388$.

doi: 10.11648/j.ajls.20140206.18

\begin{abstract}
High lipid (fats and oils) concentration contained in wastewater inhibits the activity of microbes in biological wastewater treatment systems. The lipids degradation capability of lipid-degrading bacteria was investigated for possible application in treatment of lipids-contaminated wastewater. One hundred and two bacterial isolates were isolated from 43 vegetable oil- contaminated wastewater samples of many food processing plants and restaurants in 5 districts of Can Tho city, Vietnam on LB medium. There were sixty-one isolates produced clear zones on Tw20 medium, only eleven of which were found to have the high ability to degrade vegetable oil in the contaminated wastewater. These eleven isolates were identified by PCR technique and DNA sequencing. The results of DNA sequencing were compared with GenBank database of NCBI by BLAT N software. The sequences from selected isolates showed high degrees of similarity to those of the GenBank references (between $97 \%$ and 99\%). Two isolates belonged to Bacilli (18.18\%) and nine isolates belonged to Gammaproteobacteria (81.82\%). Based on Pi value (nucleotide diversity), Gammaproteobacteria group had the highest Theta values. Theta value (per sequence) from $\mathrm{S}$ of SNP for DNA polymorphism were calculated for each group and 11 strains of lipid-degrading bacteria had high genetic diversity. The results propose Acinetobacteria soli strain AL3 a potential bioproduct for wastewater treatment because of its high ability of lipid degradation and biosafety.
\end{abstract}

Keywords: Acinetobacteria, Bacilli, Food Processing Plants and Restaurants, Lipid Contaminated Wastewater, Lipid Degradation, Vegetable Oil

\section{Introduction}

Fats, oils and greases (FOGs) are released into the environment together with wastewater derived from the food processing industry, restaurants and kitchens or by accidental spill of oils [1]. More than 400.000 tons of lipid-containing wastewater is discharged each year in Japan, and about $75 \%$ of this wastewater is from food industries and restaurants [2]. The main constituents of FOGs are animal fats and vegetable oils. They also comprise a combination of glycerol and free fatty acids whenever hydrolysis has taken place [3]. The wastewater often contains lipid such as edible oil and long-chain fatty acid [4]. Lipids present in wastewater are difficult to remove and degrade because they are difficult to dissolve in water and they are known to inhibit methanogenic processes [5]. Lipid metabolism involves several steps including emulsifying and degradation. After degradation pathway, lipid are brothen down into glycerol and fatly acid(s). The fatty acid(s) are then converted to acetyl-CoA via the beta oxidation pathway and finally enter the TCA cycle. Clogging of wastewater pipes often occurs in lipid-containing wastewater treatment systems due to the lipids present in the wastewater [6].

Many microorganisms isolated from soil and water samples have the ability to catobolize and remove wastewater lipids [7]. For environmental conservation, microbial functions have been investigated with respect to their use in treating lipid-containing wastewater [8][9]. Many studies have examined the microbial degradation of edible oils [10][11] and numerous microorganisms capable of degrading FOGs 
have been identified and may be potential candidates for bioaugmentation products.

Can Tho city is located at the central of the Mekong Delta, Vietnam with more than 1.3 million people living in 5 districts and 4 towns. This city has many food processing industries, restaurants and canteens in universities and industrial zones to serve people, students and tourists. Therefore, a big quantity of wastewater is released everyday together with an amount of lipids in wastewater. This accumulation of FOGs in wastewater collection and hardline systems leads to logging of drainpipes, appearance of unpleasant odour and corrosion of sewer pipers [12].

The aims of this study were to (i) isolate the lipid-degrading bacteria from wastewater from food processing plants and restaurants (ii) study characteristics of colonies, shape and lipid-degradation index and (iii) investigate the genetic diversity of lipid-degrading bacteria strains that have high ability of lipid-degrading in wastewater.

\section{Materials and Methods}

\subsection{Sample Collection}

Wastewater samples (1 litre/sample) were collected from wastewater drainage of many restaurants and canteen at five districts (Ninhkieu, Binhthuy, Cairang, Omon and Thotnot) of Can Tho city, Vietnam from $10^{\circ} 01^{\prime} 57^{\prime}, \mathrm{N}$ and from $105^{\circ} 47^{\prime} 03^{\prime \prime}$ E. (Figure 1). After collection, the samples were transferred immediately to the laboratory and they were stored at $5^{\circ} \mathrm{C}$.

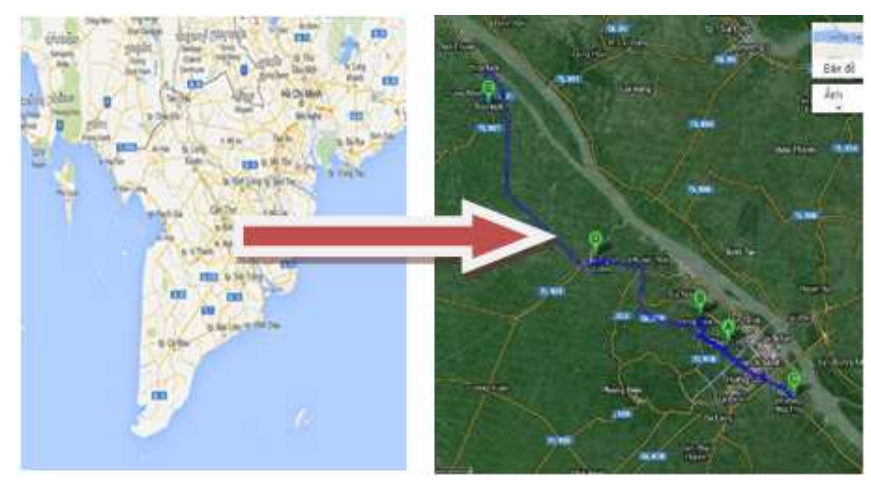

Figure 1. The geographic map showing the locations $(A, B, C, D, E)$ examined in this study, samples were collected at the five districts (Ninhkieu, Binhthuy, Cairang, Omon, Thotnot) of Can Tho city, Vietnam

\subsection{Culture Media and Growth Conditions}

Isolation media was LB [13], the screening medium [14]; Tween20 agar medium [15]. The screening medium supplemented with $1 \%$ cooking oil (Nakydaco Oil) as the natural substrate present in the wastewater from restaurants. This medium was used in this present study to detect lipid-degrading microoraganisms in the sample. The Tween 20 (Tw20)-screened isolates for degradation of the natural pollutant (cooking oil) when supplied as the only carbon and energy source in order to select the isolates that can be effectively used.

\subsection{Isolation of Lipid-Degrading Bacteria}

Ten ml sample was incubated with $200 \mathrm{ml}$ of the screening medium in flask-250mL at $30^{\circ} \mathrm{C}$ and $140 \mathrm{rpm}$ for $72 \mathrm{~h}$. After incubation, $0.5 \mathrm{ml}$ sample was spread on LB agar for single colony isolation. Bacterial colonies were differentiated bared on the basis of colony morphology and pigmentation. Colonies were subcultured on the agar-based subculture medium plates by striking technique and re-incubated at $30^{\circ} \mathrm{C}$ for 4 days. Subsequently, the isolates obtaining from the screening medium were plated on Tw20 agar and incubated at $30^{\circ} \mathrm{C}$ for 2 to 5 days. The isolates that had an opaque halo around the colonies were selected for further experiments. This isolation process was carried out in shifts of the agar-based culture medium to the agar-based subculture medium until monocultures were obtained. Monocultures were culture on the agar-based culture medium slant in the test-tube $(12 \mathrm{ml})$ and incubated at $30^{\circ} \mathrm{C}$ for 4 days following by stored $10^{\circ} \mathrm{C}$ in refrigerator.

\subsection{Colony Characteristic and Microscopic Examination}

The characteristics of colony such as size, color, shape,...etc were determined in each group. Cell morphologies of the isolates were observed using optical microscopes and scanning electron microscopes.

\subsection{Screening for Lipase Activity}

Step 1. Screening lipase activity of isolated isolates was carried out by measuring a diameter of halo zone around the isolates

A circular well (6 mm diameter) was made in each plate (Figure 2), filled with $10 \mu \mathrm{l}$ bacterial culture in LB medium, incubated at $30^{\circ} \mathrm{C}$. The diameter of each isolate was measured in the following periods: $24 \mathrm{~h}, 48 \mathrm{~h}, 72 \mathrm{~h}$ and $96 \mathrm{~h}$.
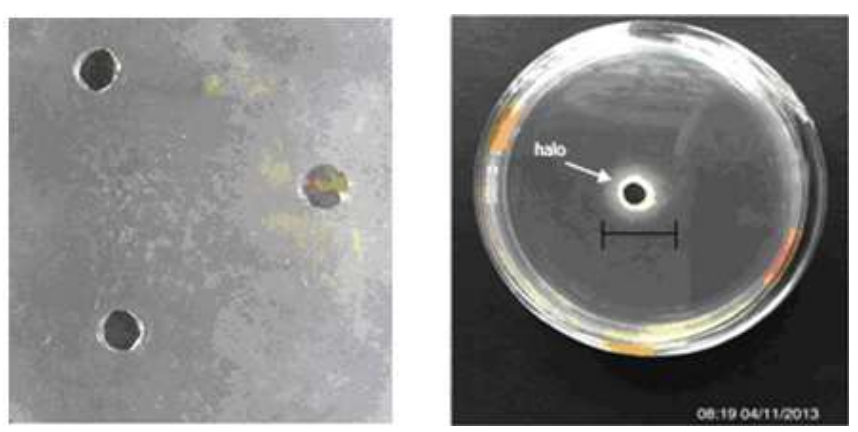

Figure 2. The wells were made on Tw20 medium agar (A) and the halo around well $(B)$

Step 2. Screening lipase activity of the selected isolates in step 1

These isolates were cultured in LB medium, $10 \mathrm{ml}$ bacterial isolates were inoculated into $200 \mathrm{ml}$ wastewater in $500-\mathrm{mL}$ plastic bottles, the treatments were incubated at $30^{\circ} \mathrm{C}$ and 100 rpm in a week, lipid concentration in the treatments were measured by Adam Rose Gottlieb method at Advanced Laboratory of Can Tho University. The experiment was completely randomized design with 3 replicates, data was 
recorded and LSD test at $\mathrm{P}=0.01$ was used to differentiate between statistically different means using SPSS version 16 .

\subsection{S rRNA Gene Amplification and Sequencing}

Bacterial DNA was isolated following published protocols [16]; The following primers were used for PCR amplification of 16S ribosomal DNA: 27F [17] and 1492R [18]. The $50 \mu \mathrm{L}$ reaction mixture consisted of $2.5 \mathrm{U}$ Taq Polymerase (Fermentas), $0.1 \mathrm{mM}$ of each desoxynecleotide triphosphate, $1.5 \mathrm{mM}$ magnesium chloride, $0.4 \mathrm{mM}$ spermidine (Sigma), 10 pM of each primer (Fermentas) and $10 \mathrm{ng}$ DNA, 10\% (vol/vol) dimethyl disulfide (Fermentas). The thermocycling cycle was carried out with an initial denaturation at $95^{\circ} \mathrm{C}(3 \mathrm{~min})$ followed by 30 cycles of denaturation at $95^{\circ} \mathrm{C}(30 \mathrm{~s})$, annealing at $55^{\circ} \mathrm{C}(30 \mathrm{~s})$, extension at $72^{\circ} \mathrm{C}(90 \mathrm{~s})$ and a final extension at $72^{\circ} \mathrm{C}$ (4 min) in C1000 Thermal Cycler (Bio-Rad) Aliquots $(10 \mu \mathrm{l})$ of PCR products were electrophoresed and visualized in $1 \%$ agarose gels using standard electrophoresis procedures. Partial 16S rRNA gene of selectived isolates was sequenced by MACROGEN, Republic of Korea (dna.macrogen.com). Finally, 16S rRNA sequence of the isolate was compared with that of other microorganisms by way BLAST; In the best isolate(s) (high ability of lipid-degrading isolates) and 10 isolates of 43 samples were chosen to sequence and the results were compared to sequences of GenBank based on partial 16S rRNA sequences to show relationships between reference strains [19]. Phylogenetic tree was constructed by the neighbor-joining method using the MEGA software version 6.06 based on 1000 bootstraps.

\subsection{SNPs Discovery}

The sequence date from 10 lipid-degrading bacterial isolates were analysed with SeqScape@Software (Applied Biosystem, Foster City, CA, USA). SeqScape is a sequence comparison tool for variant identification, SNP discovery and validation. It considers alignment depth, the base calls in each of the sequnces and the associated base quality values. Putative SNPs were accepted as true sequence variants if the quality value exceeded 20 . It means a $1 \%$ chance basecall is incorrect.

\subsection{Nucleotide Diversity $(\boldsymbol{\theta})$}

Nucleotide diversity $(\Theta)$ was calculated by the method described by Halushka et al. [20]

$$
\Theta=\mathrm{K} / \mathrm{aL} \quad \mathrm{a} \stackrel{\mathrm{n}}{=}=\sum \mathrm{V} /(\mathrm{i}-1)
$$

Where $\mathrm{K}$ is the number of SNPs identified in an alignment length, $\mathrm{n}$ is alleles and $\mathrm{L}$ is the total length of sequence (bp).

\section{Results and Discussion}

\subsection{Bacteria Isolation, Colony Characteristic and Microscopic Examination}

The lipid-degrading bacteria were developed in Tw20 medium as the previous results of Paparaskeva et al., [21] from the precipitation of free fatty acids with calcium (giving a white zone). The halo was used as an indication to detect the bacterial activity for degrading lipids and producing lipase enzymes (Table 1). Besides, Domerico et al. [22] have investigated diesel oil degrading bacteria isolated from Antarctic seawater and found that $90 \%$ of isolates had grown both diesel oil and Tween 80 .

Table 1. Total number of isolates isolated from 5 districts of Can Tho city, Vietnam

\begin{tabular}{|c|c|c|c|c|}
\hline Site (district) & $\begin{array}{c}\text { Number of wastewater } \\
\text { sample }\end{array}$ & $\begin{array}{c}\text { Isolate number were isolated } \\
\text { from LB medium }\end{array}$ & $\begin{array}{c}\text { Isolate number were isolated } \\
\text { from Tw20 medium* }\end{array}$ & $\begin{array}{c}\text { Isolate number having } \\
\text { halo around well }\end{array}$ \\
\hline Ninhkieu & 8 & 20 & 5 & 3 \\
\hline Binhthuy & 10 & 27 & 15 & 5 \\
\hline Cairang & 13 & 25 & 12 & 3 \\
\hline Omon & 6 & 15 & 14 & 0 \\
\hline Thotnot & 6 & 15 & 15 & 0 \\
\hline Total & 43 & 102 & 61 & 11 \\
\hline
\end{tabular}

They developed very well on these media from $36-48 \mathrm{~h}$ at $30^{\circ} \mathrm{C}$. Their colonies had round-shape, climy, smooth, colourless or milk-color, yellow. Some colonies appeared to have much larger size (Figure 3). The cells were observed by SEM and appeared as rod (Figure 4). They had rod shape and most of them have motility.
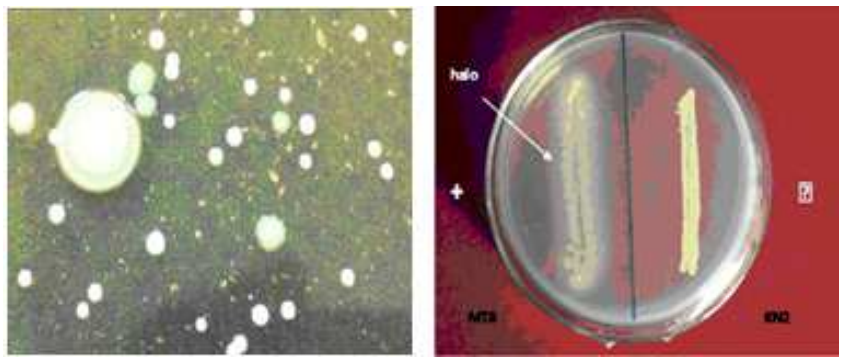

Figure 3. The colonies of several lipid-degrading isolates from wastewater (A) and the isolates having halo around their colonies 


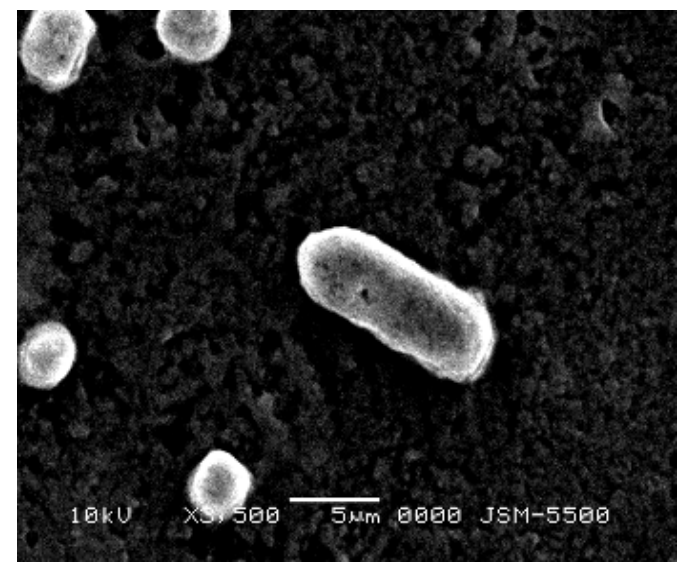

Figure 4. Electron micrographs of cell

\subsection{Screening for Lipid-Degrading Activities}

Among 102 isolates, 61 isolates had lipid-degrading activity. Only 10 isolates from 3 sites were chosen for further study (Table 1). The strains made a halo around the wells in petri agar. Isolates, AL3 and TN4B had bigger halo diameter than that of isolate TADB2 (Figure 5).
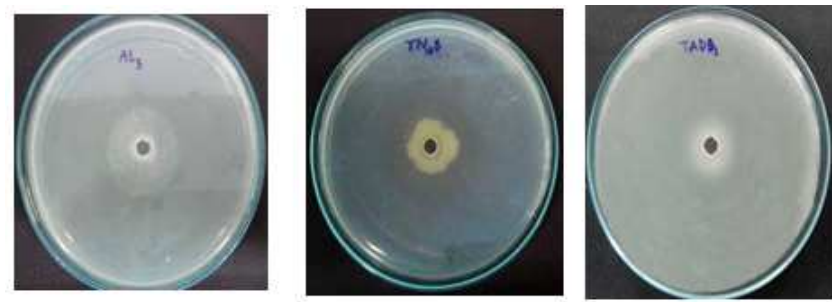

Figure 5. The isolates made the halos around the wells at central of petri dishes

The isolates had good ability of lipid degradation in wastewater in comparison with the control (Table 2) with the development of halo (big halo diameter) in 96 hours and the disappearance of lipid in the wastewater in a week (Table 3).
Table 2. The development of halo diameter $(\mathrm{mm})$ from the isolates during 96 hours on the petri-dish agar

\begin{tabular}{ccccc}
\hline & 24 hour & 48 hour & 72 hour & 96 hour \\
\hline AL1 & 12.0 & 15.6 & 18.6 & 23.6 \\
MT8 & 12.3 & 16.3 & 21.3 & 26.0 \\
MT9 & 13.3 & 20.0 & 29.0 & 40.0 \\
TADB1 & 11.3 & 21.3 & 32.2 & 35.3 \\
TN4B & 6.1 & 12.2 & 18.1 & 24.2 \\
AL3 & 13.6 & 23.3 & 27.3 & 32.4 \\
AL5 & 9.3 & 15.3 & 20.6 & 24.7 \\
AL6 & 11.1 & 16.7 & 20.1 & 24.7 \\
TD2.1 & 10.3 & 15.1 & 21.2 & 26.2 \\
TD2.3 & 11.6 & 15.3 & 20.7 & 23.1 \\
TD2.5 & 12.1 & 15.1 & 21.2 & 24.1 \\
\hline
\end{tabular}

Table 3. Lipid concentration $(\mathrm{mg} / \mathrm{l})$ in wastewater after 7 days incubation with 11 isolates and control (measured by Adam Rose Gottlied method) on shaker at $28^{\circ} \mathrm{C}$

\begin{tabular}{cccc}
\hline \multirow{2}{*}{ No } & \multirow{2}{*}{ Site } & Isolate & $\begin{array}{c}\text { Lipid concentration in wastewater } \\
(\mathbf{m g} / \mathbf{L})\end{array}$ \\
\hline 01 & Cairang & TD 2.1 & 1.694 \\
02 & & TD 2.3 & 2.702 \\
03 & & TD 2.5 & 5.447 \\
04 & \multirow{2}{*}{ Ninhkieu } & AL1 & 0.094 \\
05 & & MT8 & 0.163 \\
06 & \multirow{3}{*}{ Binhthuy } & MT9 & 0.117 \\
07 & AL3 & 0.108 \\
08 & & AL5 & 5.554 \\
09 & & AL6 & 5.345 \\
10 & & TN4B & 0.104 \\
11 & & TAD1 & 0.097 \\
& & Control & 13.486 \\
& & LSD.01 & 0.546 \\
& & C.V (\%) & 8.83 \\
\hline
\end{tabular}

\subsection{S rRNA Gene Amplification and Sequencing}

All of the 11 isolates were chosen for identification and the fragments of $1500 \mathrm{bp} 16 \mathrm{~S}$ rRNA (27F - 1492R) were obtained from PCR and sequencing (Table 4). They are lipid-degrading bacteria in wastewater.

Table 4. Phylogenetic affiliation of isolates on the basis of $16 S$ rRNA genes sequences by using BLAST programme in the GenBank database based on sequence similarity

\begin{tabular}{|c|c|c|}
\hline Taxonomic group and strain & Closest species relative & Similarity $(\%)$ \\
\hline \multicolumn{3}{|l|}{ Gammaproteobacteria } \\
\hline AL1 & Acinetobacter baumannii IARI-JR-51 (KF055001) & 99 \\
\hline AL3 & Acinetobacter soli strain KZ-1 (JX499235) & 98 \\
\hline TD2.1 & Acinetobacter ursingii, strain: MTCC 9826 (AB859677) & 97 \\
\hline TD2.5 & Acinetobacter septicus strain BA9 (FJ263921) & 97 \\
\hline MT9 & Acinetobacter bereziniate strain JDG127 (JX035952) & 99 \\
\hline TADB1 & Pseudomonas hibiscicola strain R4-721 (JQ659711) & 97 \\
\hline AL5 & Aeromonas hydrophila strain A-X2B (KJ806436) & 99 \\
\hline AL6 & Aeromonas media strain JHS07 (GU205201) & 99 \\
\hline TN4B & Stenotrophomonas maltophilia, isolate AAIII-3 (LN558616) & 99 \\
\hline \multicolumn{3}{|l|}{ Bacilli } \\
\hline TD2.3 & Bacillus pumilus strain ChST1.7 (JF935095) & 99 \\
\hline MT8 & Bacillus cereus DBT3ST4 (GU122949) & 98 \\
\hline
\end{tabular}



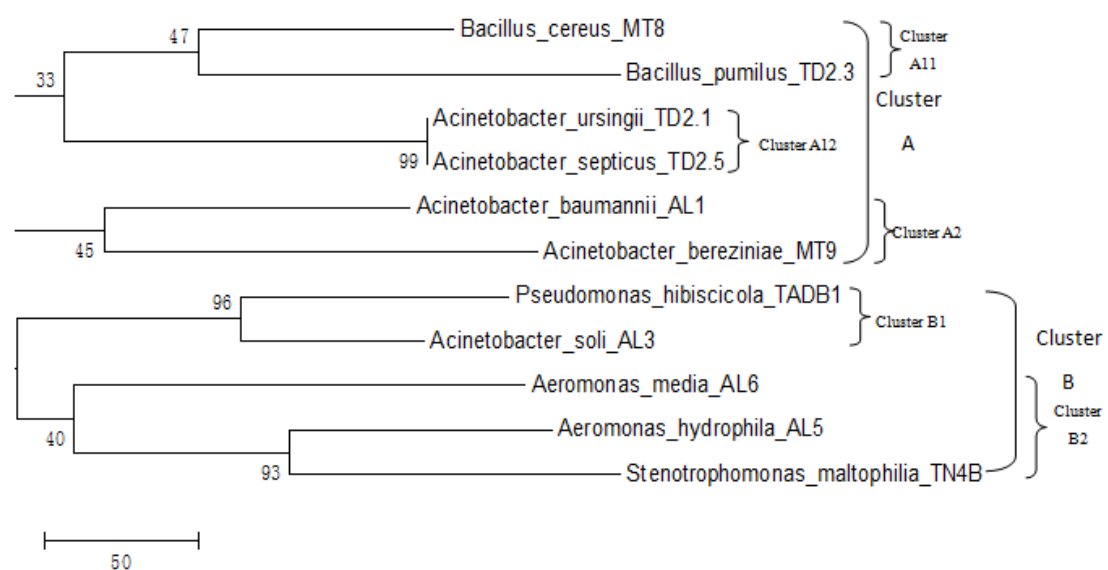

Figure 6. Phylogenetic tree for partial $16 S$ rRNA gene sequences from 11 isolates by using primers $(27 F, 1492 R)$ showing relationships between presented strains along with related sequences retrieved from GenBank. The numbers at the nods indicate the levels of bootstrap support (\%) based on a Neighbor-Joining analysis of 100 re-sampled datasets. The scale bar indicates the phylogenetic distance corresponding to 5 changes per 100 bases.

The determination of nearest neighbor phylogenetic sequences (for 16S rRNA gene sequences) of the 11 isolates (by the BLAST search program) showed that they grouped into two clusters (Figure 6). Cluster A divided two small cluters: cluster A1 composed of cluster A11 with Bacillus cereus MT8 and Bacillus pumilus TD2.3; cluster A12 with Acinetobacter usingii TD2.1 and Acinetobacter septicus TD2.5, and cluster A2 with Acinetobacter baumanii AL1 and Acinetobacter beneziniae MT9.

Cluster B comprised two clusters: Cluster B1 had two strains Pseudomonas hibiscicola TADB1 and Acinetobacter soli AL3; Cluster B2 had two branches including Aeromonas media AL6 and Aeromonas hydrophila AL together with Stenophomonas maltophila TN4B. Three strains had close relationship. This result showed that Gram-positive bacteria (two strains Bacillus cereus and Bacillus pumilis) (in a separate branch) and Gram-negative bacteria (with the bacterial strains) were clustered in different branches. Eleven strain belonged to the class Bacilli (18.18\%) and Gamma-Proteobacteria occupied $81.82 \%$ composing of genus Acinetobacter (55.55\%), Aeromonas (22.22\%), Pseudomonas $(11.11 \%)$ and Stenotrophomonas (11.11\%)(Figure 7).
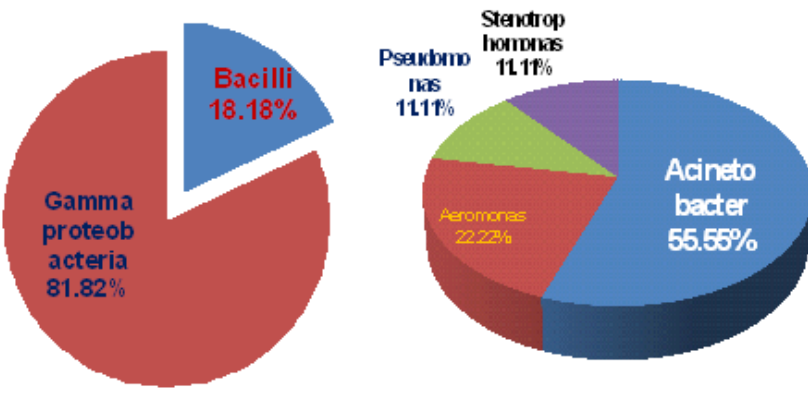

Figure 7. The proportion of bacterial groups

Nucleotide polymorphism can be measured by many methods, for example, halotype (gene) diversity, nucleotide diversity, (Pi), Theta $(\Theta)$ (per group) etc... In this study, nucleotide diversity was estimated as Theta $(\Theta)$, the number of segregating sites [23], and its standard deviation (SO).

These parameters were estimated by DNA Sequence Polymorphism software version 4.0 [24]. Pi value explained nucleotide diversity of sequences for each gene; the higher values, the more diversity among. Proteobacteria group had the highest values and Bacilli group had the lowest values. Theta values (per sequence) from $\mathrm{S}$ of SNP for DNA polymorphism were calculated for each group and lipid-degrading bacteria group had the high values especially different nucleotides even though 11 straind were isolated in wastewater from food processing plants and restaurants of 3 in 5 districts of Can Tho city (Table 5).

Table 5. Genetic diversity of 11 strains

\begin{tabular}{cccc}
\hline & $\begin{array}{c}\text { Nucleotide } \\
\text { diversity }\end{array}$ & $\begin{array}{c}\text { Theta (per site) } \\
\text { from Eta }\end{array}$ & $\begin{array}{c}\text { Theta (per site) } \\
\text { from S }(\boldsymbol{\theta})\end{array}$ \\
\hline \multicolumn{1}{|c|}{ 11 strains } & 0.72932 & $0.942 \pm 0.134$ & $0.341 \pm 0.010$ \\
Primer 27F & 5'- AGA GTT TGA TCC TGG CTC - 3' \\
Primer 1492R & 5'- GGC TAC CTT TGT TAC GAC TT - 3' \\
\hline
\end{tabular}

Ruis et al [25] isolated a strain Bacillus sp. CR-179 from subtropical forest soil of Puerto Iguazu (Argentina) which degraded lipid and polysaccaride in wastewater and El-Bestawy et al. [15]. Eight bacterial species were isolated from vegetable oil and grease-contaminated industrial wastewater. However, only four of which were found to have the ability to degrade oil and grease in the contaminated wastewater. These isolates were identified according to morphological and biochemical profiles as Pseudomonas and E. coli (gram-negative bacteria) and Cappelo et al. [26] identified two oil-degrading bacteria strains (BW-1 and BW-2) from Bilge water, were clustered with Acinetobacter genus (a similarity of $100 \%$ and $99 \%$, respectively). While Sugimori et al. [7] isolated a strain of bacterium Raoultella planticola strain 232-2 that is capable of efficiently catabolozing lipids under acidic conditions such as in grease traps in restaurants and food processing plants. Furthermore, Sugimori and Utsue [4] also isolated two bacterial strains with high degradation abilities at an alkaline $\mathrm{pH}$ from Japanese soil, as Acinetobacteria sp. strain SS-192 and Pseudomonas aerunosa 
strains SS-219. Our results isolated and identified 11 lipid-degrading bacterial strains with two groups: Gammaproteobactera and Bacilli. Gammaproteobacteria composed of Acinetobacteria, Pseudomonas, Aeromonas and Stenotrophomonas with high ratio in comparison to Bacilli with two species (Bacillus cereus and Bacillus pumilus) however these strains have been known as pathogenic bacteria.

Based on bio-safety and high lipid degradation ability, this study selected a strain as Acinetobacter soli to evaluate its ability on lipid degradation in wastewater in vitro and the big volume in 100 or $1000-\mathrm{L}$ containers.

\section{Conclusion}

From 43 wastewater samples in food processing plants and restaurants in 5 districts of Can Tho city, Vietnam, 102 isolates were isolated on LD medium and 61 isolates on Tw20 medium. Finally, 11 isolates having high lipid degradation ability were chosen to analyse their relationship and they showed that bacterial diversity was very high and $1 / 11$ strains was suggested to produce bioproducts for lipid degradation in wastewater treatment in big models.

\section{Acknowledgements}

The authors thank the help of Biology BSc. Students and technicians in the Environment Microbiology Laboratory, Biotechnology R\&D Institute, Can Tho University, Vietnam, especially Associate Professor Dr. TRUONG TRONG NGON, Head of Molecular Biology Department, Biotechnology R\&D Institute, Can Tho University, Vietnam analysed data and nucleotide diversity.

\section{References}

[1] V. Cipinyte, S. Grigiskis and E. Baskys, "Selection of fat-degrading microorganisms for the treatment of lipid-contaminated environment," Biologija, , vol. 35, pp. 84-92, 2009.

[2] M. Yada, K. Kawakuchi, and Y. Mihara, "Haikibutsu no bioconversion," Bioconversion of wastes, Chijin Shokan, Tokyo, 2001.

[3] M.C. Cammarota, G.A. Teixeira, and D.M.G. Freire, "Enzymatic pre-hydrolysis and anaerobic degradation of wastewaters with high fat contents," BioTechnol. Letters, vol.23, pp.1591-1595, 2001.

[4] D. Sugimori, and T. Utsue, "A study of the efficiency of edible oils degraded in alkaline conditions by Pseudomonas aeruginosa SS-219 and Acinetobacter sp. SS-192 bacteria isolated from Japanese soil," World J. Microbiol. Biotechnol., vol. 28(3), pp.841-848, 2013. doi:10.1007/s11274-011-0880-6.

[5] M. Hatamoto, H. Imachi, Y. Yashiro, A. Ohashi and H. Harada, "Diversity of Anaerobic Microorganisms Involved in Long-Chain Fatty Acid Degradation in Methanogenic Sludges as Revealved by RNA-Based Stable isotope Probing," Appl. And Environ. Microbiology, vol. 73(13), pp.4119-4127, 2007.
[6] D. Sugimori, M. Nakamura, and M. Mihara, "Microbial degradation of lipid by Acinetobacter sp. strain SOD-1," Biosci. Biotechnol. Biochem, vol.66(7), pp.1579-1582, 2002.

[7] D. Sugimori, M. Watanabe and T. Utsue, "Isolation and lipid degradation profile of Raoultella planticola strain 232-2 capable of efficiently catabolozing edible oils under acidic conditions,” Appl. Microbiol. Biotechnol., vol. 97, pp;871-880, 2013.

[8] J.P. Canler, C. Roger and Ph. Duchene, "Aerobic biological treatment of grease from urban wastewater treatment plants," Water Sci. Technol., vol. 44, pp.219-226, 2001.

[9] Y. Wei, R.T.V. Houten, A.R. Borger, D.H. Eikelboom, and Y. Fan, "Minimization of excess sludge production for biological wastewater treatment," Water Res., vol. 37, pp.4453-4467, 2003.

[10] Y. Yamaoka, K. Takeno, H. Shinkawa, N. Noparatnaraporn and K. Sasaki, "Isolation of a thermotolerant photosynthetic bacterium, Rhodobacter spaeroides strain, NAT, and its capasity for oil and chemical oxygen demand removal at high temperatures," Biosci. Biotechnol. Biochem., vol. 72(6), pp.1601-1603, 2008.

[11] D, Tanaka, M. Takashima, M. Mizuta, S Tanaka, A. Sakatoku, A. Nishikawa, T. Osawa, M. Noguchi, S.I. aizawa and S. Nakamura, "Acinetobacter sp. Ud-4 efficiently degrades both edible and mineral oils: Isolation and charactization," Curr. Microbiol, vol. 60(3), pp.203-209, 2010.

[12] N.F. Gray, "Biology of wastewater treatment," Oxford University press, pp.6-38, 1989.

[13] S Bennasar A., C. Guasp and J. Lalucat, "Molecular methods for the detection and identification of Pseudomonas stutzeri in pure culture and environmental samples," Microbiol. Ecol., vol.35, pp:22-33, 1998.

[14] Y. Matsumiya, D. Wakita, A. Kimura, S. Sanpa, and M. Kubo, "Isolation and characterization of a lipid-degrading bacterium and its application to lipid-contaning wastewater treatment," J. Biosci. Bioeng., vol 103(4), pp. 325-330, 2007.

[15] E. El-Bestawy, M. H. El-Masry and N. El-Adl, "The potentiality of free Gram negative bacteria for removing oil and grease from containing industrial effluents," World Journal of Microbiology and Biotechnology, vol. 21, pp:815-822, 2005.

[16] B. Neumann, A. Pospiech, and H.U. Schairrer, "Rapid isolation of genomic DNA from Gram-negative," Trends Gent., vol. 8, pp. 332-333, 1992.

[17] D.J. Lane, "16S/23S rRNA sequencing," Nucleic acid techniques in bacterial systematics, pp. 115-175. Edited by E. Stackebrandt and M.Goodfellow. NewYork: John Wiley, 1991.

[18] S. Turner, K.M. Pryer, V.P.W. Miao and J.D. Palmer, "Investigating deep phylogenetic relationships among cyanobacteria and plastids by small subunit rRNA sequence analysis," Journal of Eukaryotic Microbiology, vol.46, pp:327-338, 1999.

[19] K. Tamura, D. Peterson, N. Peterson, G. Stecher, M. Nei, and S. Kumar, "MEGA5: Molecular Evolutionary Genetics Analysis using Maximum Likehood, Evolutionary Distance and Maximum Parsimony Methods," Mol. Biol. Evol., vol. 28, pp. 2731-2739, 2011. 
[20] M.K. Halushka, J.B. Fan, K. Bentley, L. Hsie, N. Shen, A. Weder, R. Cooper, R. Lipshutz, and A. Charavarti, "Patterns of single-nucleotide polymorphisms in candidate genes for blood-pressure homestasis," Nat. Genet., vol. 22(3), pp. 239-247, 1999.

[21] D. Paparaskeas, P. Christakopoulas, D. Kekos, and J.B. Marcis, "Optimization production of extracellular lipase from Rhodorula glutinis," Biotechnology Letters, vol. 1, pp. 397-402, 1992.

[22] M. Domenico, A. Guidice, L. Michaud, Pollar Res., vol. 23(2), pp.141-161, 2004.

[23] G.A. Watterson, "On the number of segregation sites in general models without recobination," Theor. Pop. Biol., vol. 7, pp. 256-276, 1975.
[24] J. Rozas, and R. Rozas, "DnaSP version 4.1: an integrated program for molecular population genetics and molecular evolution analysis," Bioinformatics, vol. 15, pp. 174-175, 2005.

[25] C. Ruiz, F.I.J. Pastor and P. Diaz, "Isolation of lipid- and polysaccharide-degrading microorganisms from subtropical forest soil, and analysis of lipolytic strain Bacillus sp. CR-179," Letters in Applied Microbiology, vol. 40, pp:218-227, 2005.

[26] S. Cappello, S. Santisi, R. Calogero, M. Hassanshahian, M.M. Yakimov, "Charactisation of Oil-Degrading Bacteria Isolated from Bilge Water," Water Air Soil Pollut., vol. 223, pp:3219-3226, 2012. 